\title{
Design Charts to Maximize the Gain-Bandwidth Product of Capacitive Micromachined Ultrasonic Transducers
}

\author{
Selim Olcum, Muhammed N. Senlik, Can Bayram and Abdullah Atalar \\ Dept. of Electrical and Electronics Engineering \\ Bilkent University \\ Ankara, Turkey 06800 \\ Email: selim@ee.bilkent.edu.tr
}

\begin{abstract}
In this work we define a performance measure for capacitive micromachined ultrasonic transducers (cMUT) in the form of a gain-bandwidth product to investigate the conditions that optimize the gain and bandwidth with respect to device dimensions, electrode size and electrical termination resistance. For the transmit mode, we define the figure of merit as the pressurebandwidth product. Fully-metallized membranes achieve a higher pressure-bandwidth product compared to partially metallized ones. It is shown that the bandwidth is not affected by the electrode size in the transmit mode. In the receive mode, we define the figure of merit as the gain-bandwidth product. We show in this case that the figure of merit can be maximized by optimizing the electrode radius. We present normalized charts for designing an optimum cMUT cell at the desired frequency with a given bandwidth for transmit or receive modes. The effect of spurious capacitance and liquid loading effect are considered. Design examples are given to clarify the use of these charts.
\end{abstract}

\section{INTRODUCTION}

It is shown that a large bandwidth is possible with an untuned cMUT immersed in water [1], [2]. For such a cMUT, the operation frequency range may extend from very low frequencies to the antiresonance of the membrane [3]. However, those cMUTs have small conversion efficiencies and are not as sensitive as piezoelectric transducers. In this work, we explore the limits of a cMUT operating in different regimes using the Mason model corrected with finite element method (FEM) simulations. We try to maximize the bandwidth of a cMUT while keeping the output pressure or the conversion efficiency at a reasonable value. For this purpose, we define performance measures in the form of a pressure-bandwidth product or a gain-bandwidth product. We try to maximize this figure of merit by optimizing various geometrical parameters of the cMUT.

\section{MASON MODEL}

Commonly, a numerical analysis of cMUTs is based on the Mason's equivalent circuit model. This lumped model has been utilized in many studies before [1], [2]. The equivalent circuits of a cMUT in transmit (a) and in receive (b) modes are demonstrated in Fig. 1, where $C_{0}$ is the capacitance between the electrodes, $C_{S}$ is the parallel spurious capacitance, $n$ is the turns ratio, $Z_{m}$ is the lumped mechanical impedance

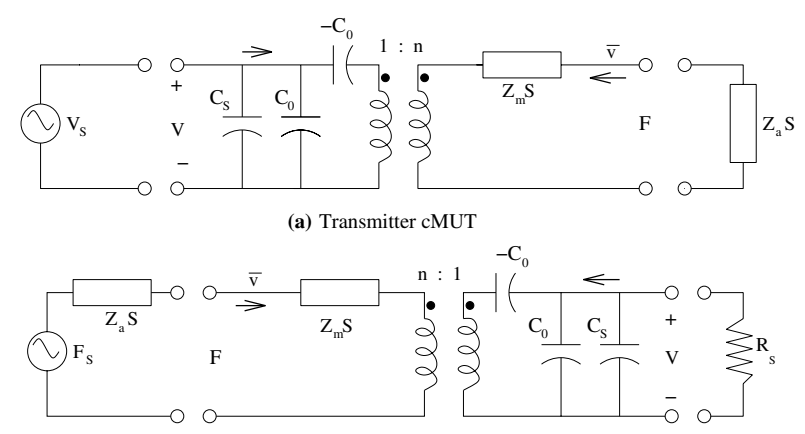

(b) Receiver cMUT

Fig. 1. Mason model (a) for a cMUT operating as a transmitter excited by a voltage source $\left(V_{S}\right)$ to drive the acoustic impedance of the immersion medium $\left(Z_{a} S\right)$, (b) for a cMUT operating as a receiver excited by the acoustical source $\left(F_{S}, Z_{a} S\right)$ to drive the electrical load resistance of the receiver circuitry $\left(R_{S}\right)$.

of the membrane, $S$ is the membrane area and $Z_{a}$ is the acoustical impedance of the immersion medium. A negative series capacitance $-C_{0}$ is included to take the spring softening effect into account. Note that, for the receive mode equivalent circuit, the electrical side is terminated with an electrical termination resistance, $R_{S}$.

The Mason Model formulations used in this paper are based on the model depicted in [4]. The differences are demonstrated below. The circuit parameters are calculated both using simple numerical calculations ${ }^{1}$ and FEM simulations ${ }^{2}$. Additionally, the effect of water loading is included in the analysis using the method in [5].

To be able to consider partial electrode cases, the turns ratio, $n$ is calculated using the method developed in [6]:

$$
n=K \frac{F_{\text {effective }}}{V_{A C}}
$$

where $\mathrm{K}$ is a lumped correction factor given by $K=0.58 \pm$ 0.05 .

The collapse voltage of the membrane is calculated using the method developed in [7]. An approximate formula is given

\footnotetext{
${ }^{1}$ Numerical calculations are performed using MATLAB

${ }^{2}$ FEM simulations are performed using ANSYS
} 


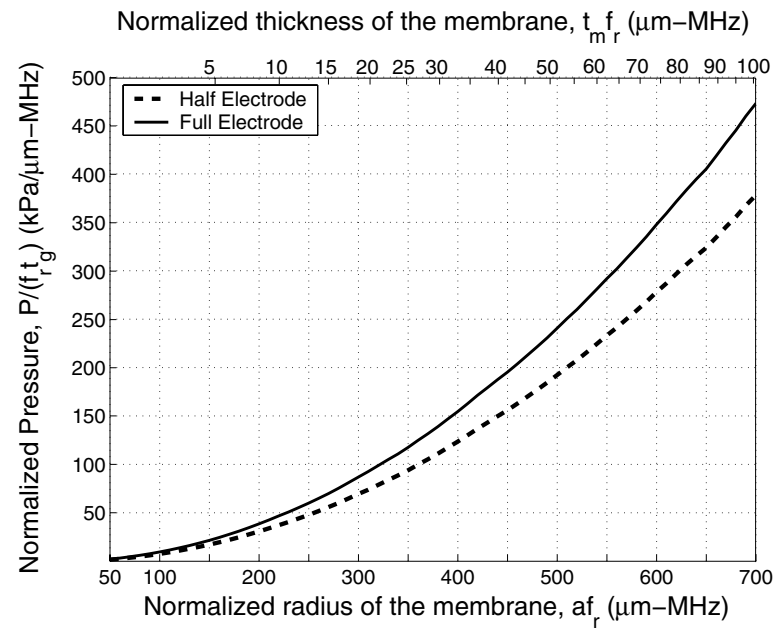

Fig. 2. Normalized pressure as a function of normalized membrane radius or thickness for transmitter cMUTs with full metallized (solid), or half metallized membranes (dashed).

below for design purposes:

$$
V_{c o l} \simeq \gamma \sqrt{\frac{128\left(Y_{0}+T\right) t_{m}^{3} \bar{t}_{g}^{3}}{27 \epsilon_{0}\left(1-\sigma^{2}\right) a^{4}}}
$$

where $\gamma$ is equal to 0.7 and 0.82 for full metallized and half metallized membranes, respectively.

\section{Performance Optimization}

The performance of a transducer can be maximized by optimizing the membrane radius $(a)$, membrane thickness $\left(t_{m}\right)$, gap thickness $\left(t_{g}\right)$, electrode radius and the electrical termination resistance $\left(R_{S}\right)$. In order to make a fair comparison, we always keep the maximum applied bias voltage at the $90 \%$ of $V_{c o l}$. Furthermore, we compare transducers with equal natural resonance frequency, $f_{r}$.

\section{A. Transmit Mode}

In the transmit mode, there is no electrical limitation on the applied voltage other than the collapse voltage of the membrane or the electrical break-down of the insulation material. The electrical mismatch between the electrical source and the transducer is not a concern. The produced pressure at the output port is the important parameter along with the 3$\mathrm{dB}$ bandwidth of the output pressure, $B_{1}$. Referring to the Fig. 1(a), we define $P$ as the pressure in the immersion medium, $P=F / S$, when the applied AC voltage, $V_{S}$ is at the maximum allowable value. Therefore, we define the figure of merit for transmit mode as:

$$
M_{T}=P B_{1}
$$

\section{B. Receive Mode}

In the case of receive mode, the input signal power is limited. Therefore, we should utilize the available acoustical power from the source as much as possible. The mismatch losses both at the acoustical side and at the electrical side

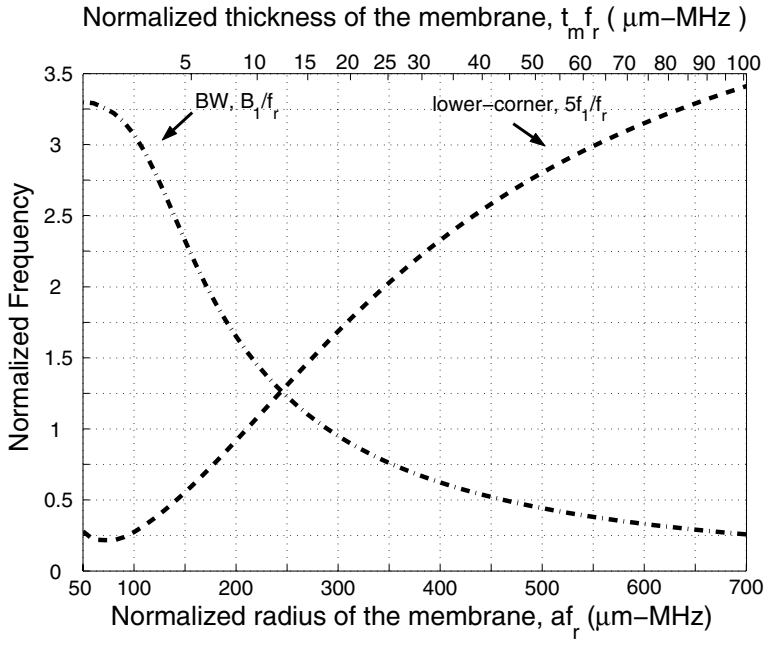

Fig. 3. Normalized bandwidth (dash-dot) and lower corner frequency (dashed) as a function of normalized membrane radius or thickness for transmitter cMUTs.

must be minimized for the maximum performance. Transducer power gain, $G_{T}$, (the ratio of power delivered to electrical load to the power available from the source) [8] takes into account the mismatch losses for both input and output ports. Using $\sqrt{G_{T}}$ as gain ${ }^{3}$ and $B_{2}$ as the 3-dB bandwidth of the gain, we define the gain-bandwidth product as

$$
M_{R}=\sqrt{G_{T}} B_{2}
$$

\section{Design Graphs}

Utilizing the figure of merit definitions, the performance charts of cMUTs for different $a$ and $t_{m}$ values are produced. While sweeping $a, t_{m}$ is also varied in order to keep $f_{r}$ constant. Initially, $C_{S}$ is kept at zero. It was shown in [4] that the performance of the transducers can be normalized with respect to $f_{r}$ and $t_{g}$. In the following charts, all the axes are normalized and their relation with the actual values is provided in the axis labels.

For simplicity we treat the problem as if it is linear, although a cMUT is a highly nonlinear device. The resulting normalized pressure and bandwidth figures are seen in Figs. 2 and 3. We also show the effect of reducing the radius of electrode metallization by a factor of 2 . Notice the trade off between the increasing pressure, $P$ and decreasing bandwidth, $B_{1}$ as $a f_{r}$ increases. The resulting $M_{T}$ is normalized and plotted in Fig. 4.

For the receive mode of operation, the applied bias voltage is kept constant at $90 \%$ of the collapse voltage. In this case, the figure of merit, $M_{R}$, is independent of the gap height, $t_{g}$ [4]. The results of the receive mode simulations are presented in Figs. 5, 6 and 7. Note that $R_{S}$ is optimally chosen for each $a-t_{m}$ pair and the normalized value of $R_{S}$ is plotted in Fig. 8 .

We consider the effect of the spurious capacitance, $C_{S}$ in Fig. 9. It is clear that existence of $C_{S}$ reduces both the gain and bandwidth.

\footnotetext{
${ }^{3}$ Square root of $G_{T}$ is used to get a voltage based gain.
} 


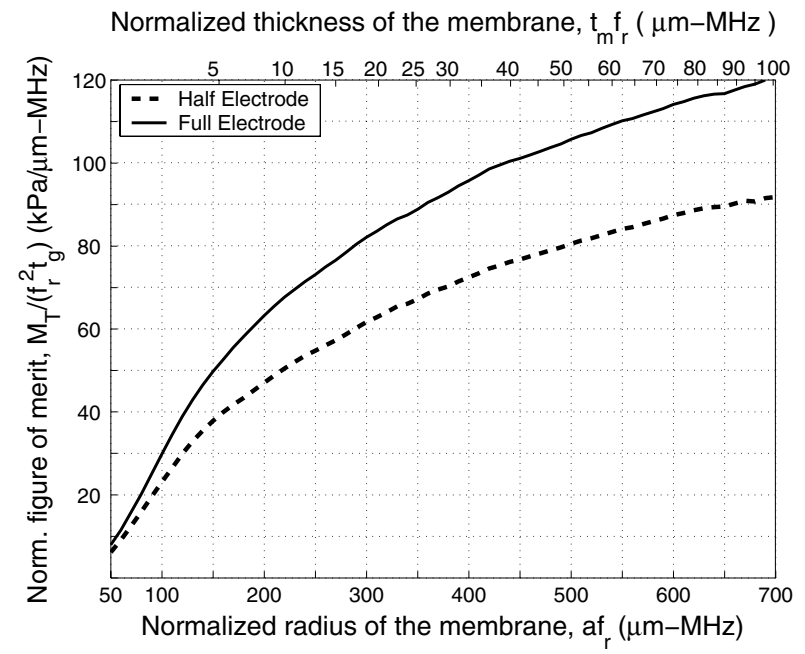

Fig. 4. Normalized pressure-bandwidth product as a function of normalized membrane radius or thickness for transmitter cMUTs with full metallized (solid), or half metallized membranes (dashed).

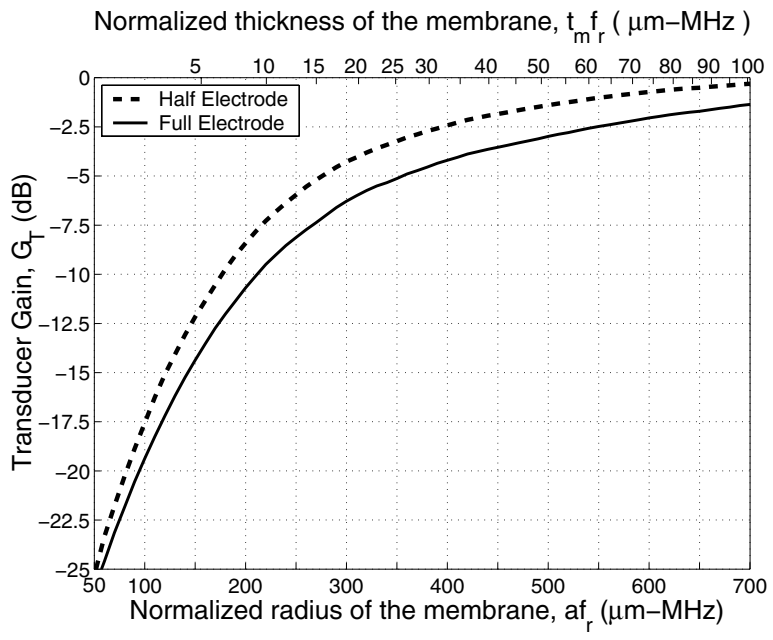

Fig. 5. Normalized transducer gain as a function of normalized membrane radius or thickness for receiver cMUTs with full metallized (solid), or half metallized membranes (dashed). The curves are independent of $t_{g} . C_{S}=0$.

\section{Design EXAmples}

Let us demonstrate the use of the graphs by designing a transmitter cMUT operating between the 3 -dB frequencies $f_{1}$ to $f_{2}$ with an output pressure per voltage as high as possible. Suppose $f_{1}=1 \mathrm{MHz}$ and $f_{2}=15 \mathrm{MHz}$, meaning $B_{1}=14 \mathrm{MHz}$. We first pick the point where $a f_{r}=200 \mu \mathrm{m}-\mathrm{MHz}$ in Fig. 3. $B_{1} / f_{r}=1.65$ at this point implies $f_{r}=8.5 \mathrm{MHz}$. We read $5 f_{1} / f_{r}=0.9$, resulting $f_{1}=1.5 \mathrm{MHz}$ which is larger than the $1 \mathrm{MHz}$ requirement. After a few iterations we find $a f_{r}=175$ satisfies the specifications. In this case, $B_{1} / f_{r}=1.9$ so $f_{r}$ is $7.4 \mathrm{MHz}$ and $5 f_{1} / f_{r}=0.7$ with $f_{1} \simeq 1 \mathrm{MHz}$. We complete the design by calculating other parameters. The required transducer radius is $175 / 7.4 \simeq 24 \mu \mathrm{m}$. From the upper $\mathrm{x}$-axis of Fig. 3, we determine the thickness, $6.4 / 7.4 \simeq$ $t_{m}=0.9 \mu \mathrm{m}$. To achieve a high output pressure we should pick

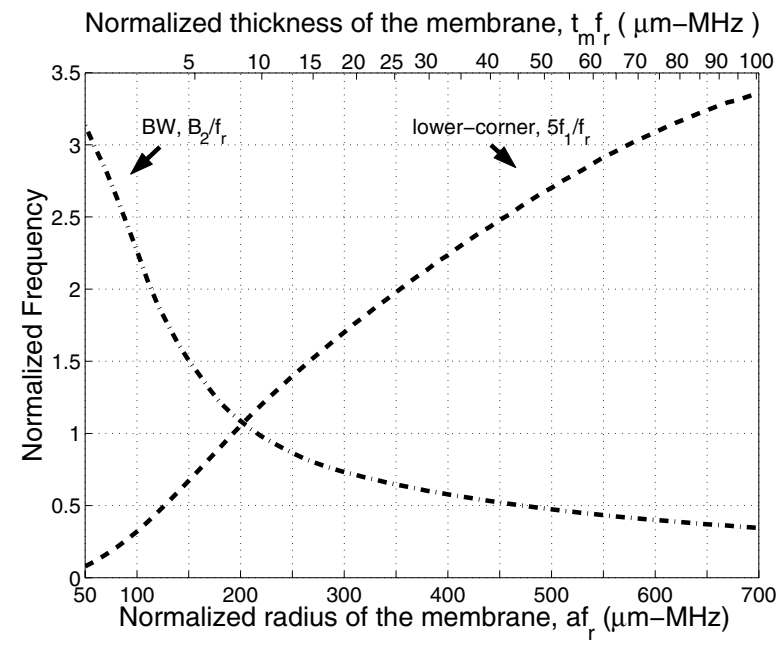

Fig. 6. Normalized bandwidth (dash-dotted) and lower corner frequency (dashed) as a function of normalized membrane radius or thickness for receiver cMUTs with full metallized or half metallized membranes. The curves are independent of $t_{g} . C_{S}=0$.

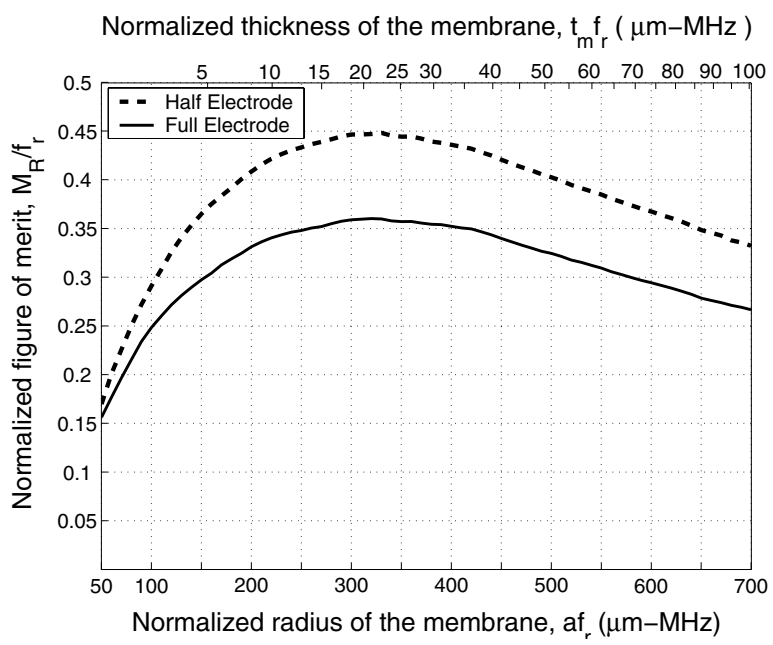

Fig. 7. Normalized gain-bandwidth product as a function of normalized membrane radius or thickness for receiver cMUTs with full metallized (solid), or half metallized membranes (dashed). The curves are independent of $t_{g}$. $C_{S}=0$.

the collapse voltage as high as possible. Let us assume we have a voltage source that can generate pulses up to $150 \mathrm{~V}$. Hence, if $V_{c o l}=150 \mathrm{~V}$, from Eq. 2, effective $t_{g}$ value would be calculated as $\simeq 0.5 \mu \mathrm{m}$. Using Fig. 4 the figure of merit is calculated as $58 \times 7.2^{2} \times 0.5 \simeq 1500 \mathrm{kPa}-\mathrm{MHz}$. Since the bandwidth is $14 \mathrm{MHz}$, the output pressure $P \simeq 105 \mathrm{kPa}$.

Suppose we need a cMUT with an output pressure of $300 \mathrm{kPa}$ with a center frequency of $6 \mathrm{MHz}$. Let's use the design graphs to determine the device dimensions. If we choose $t_{g}=0.5 \mu \mathrm{m}$ and $f_{r}=6 \mathrm{MHz}$, we find $P /\left(f_{r} t_{g}\right)=$ $300 /(6 \times 0.5)=100$. Using Fig. 2 we determine $a f_{r}=320$ and $t_{m} f_{r}=21$ resulting $a=53 \mu \mathrm{m}$ and $t_{m}=3.5 \mu \mathrm{m}$. The estimated collapse voltage (Eq. 2) is calculated as $250 \mathrm{~V}$. From Fig. 3 the bandwidth $B_{1}=0.85 \times 6=5.1 \mathrm{MHz}$ and 


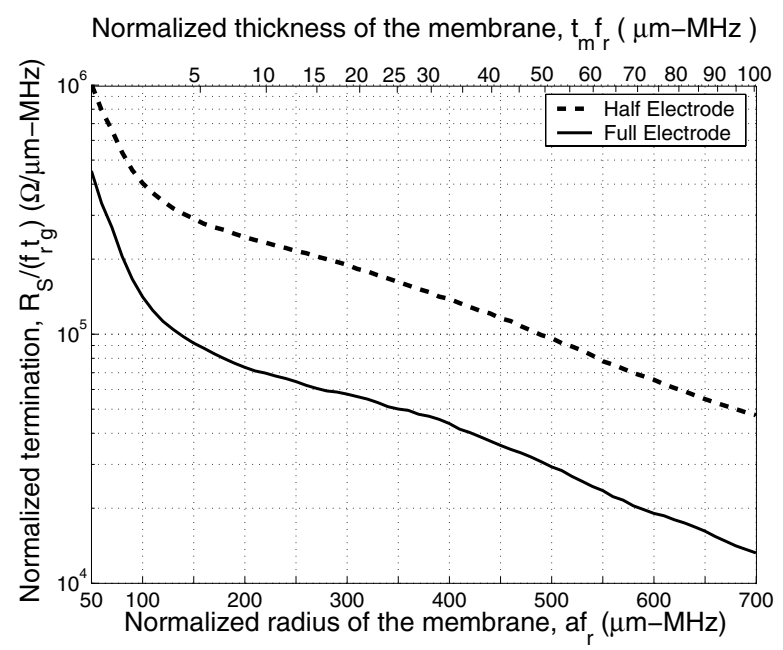

Fig. 8. Normalized termination resistance, $R_{S}$ as a function of normalized membrane radius or thickness for receiver cMUTs with full metallized (solid), or half metallized membranes (dashed). $C_{S}=0$.

$f_{1}=1.85 \times 6 / 5=2.2 \mathrm{MHz}$.

As an example for designing a receiver cMUT, suppose we need $10 \mathrm{MHz}$ bandwidth between $2 \mathrm{MHz}$ and $12 \mathrm{MHz}$ 3 - $\mathrm{dB}$ corner frequencies. We decide to fabricate our transducers with a half top electrode, since we wish a higher transducer gain. Using the dashed curves we read $B_{2} / f_{r}=0.75$ for $a f_{r}=300 \mu \mathrm{m}-\mathrm{MHz}$. In this case $f_{r}$ should be $13.4 \mathrm{MHz}$. For this choice, $f_{1}$ is calculated from $5 f_{1} / f_{r}=1.65$ as $4.4 \mathrm{MHz}$ which is above the $2 \mathrm{MHz}$ requirement. After a few iterations we determine that when $a f_{r}=200 \mu \mathrm{m}-\mathrm{MHz}, f_{r}$ is $9 \mathrm{MHz}$ and it satisfies $B_{2}=10 \mathrm{MHz}$ and $f_{1}=2 \mathrm{MHz}$. Therefore, $a$ should be $22.5 \mu \mathrm{m}$ and $t_{m}$ should be $0.9 \mu \mathrm{m}$. The transducer gain is determined from Fig. 5 as $-8.5 \mathrm{~dB}$. In order to achieve an acceptable bias voltage, we choose $t_{g}=0.25 \mu \mathrm{m}$. In this case, $V_{\text {col }}$ is calculated as $\simeq 77 \mathrm{~V}$. The termination resistance should be $230 \mathrm{~K} \times 0.25 \times 9 \simeq 520 \mathrm{~K} \Omega$ per transducer. Therefore, if 104 cMUTs are connected in parallel, an electrical load of $5 \mathrm{~K} \Omega$ is necessary.

As our last example let us suppose that we want to design a transducer with a transducer gain of $-3 \mathrm{~dB}$ centered at $8 \mathrm{MHz}$. Utilizing Fig. 5, we determine when $a f_{r}=360$ the gain requirement is satisfied. At this point $B_{2} / f_{r}=0.65$ and $5 f_{1} / f_{r}=2$ (Fig. 6) . In order to achieve $f_{1}+B_{2} / 2=8 \mathrm{MHz}$, we set $2 f_{r} / 5+0.65 f_{r} / 2=8 \mathrm{MHz}$ or $f_{r}=11 \mathrm{MHz}$ and $f_{1}=4.4 \mathrm{MHz}$. Since we determined $f_{r}, a \simeq 33 \mu \mathrm{m}, t_{m}=2.6 \mu \mathrm{m}$. For a gap height of $0.25 \mu \mathrm{m}$ collapse voltage is calculated as $\simeq 160 \mathrm{~V}$. In this case, the electrical termination resistance per cell should be $150 \mathrm{~K} \times 0.25 \times 11 \simeq 410 \mathrm{~K} \Omega$.

\section{CONCLUSION}

We defined performance measures for cMUTs in transmit and receive modes. We presented ways of maximizing these measures considering both gain and bandwidth by optimizing the geometrical and electrical parameters. For the transmit mode, larger gap heights and electrode sizes are preferable,

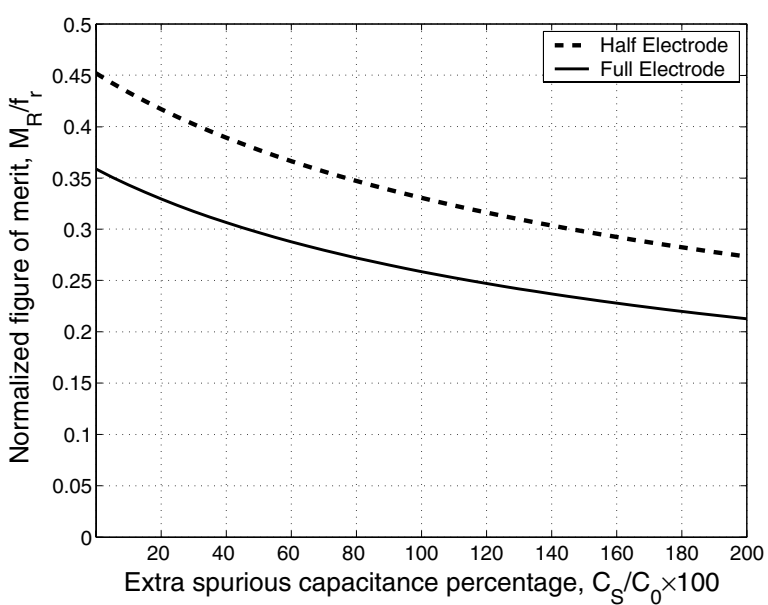

Fig. 9. Maximum value of normalized gain-bandwidth product as a function of spurious capacitance, $C_{S}$, with respect to the shunt input capacitance for receiver cMUTs with full metallized (solid), or half metallized membranes (dashed).

since higher collapse voltages and turns ratios are possible. Additionally, smaller membrane radii result in higher bandwidth at the expense of pressure and pressure-bandwidth product. For the receive mode, the gap height does not effect the performance. Half metallized membranes are optimum if spurious capacitors are negligible. Additionally, there is an optimal value for radius or thickness and electrical termination resistance for a given resonance frequency. For very high bandwidth values, gain and the gain-bandwidth product must be sacrificed. An electrical spurious capacitor is detrimental for the performance of cMUT in receive mode. We introduce design tools to determine the optimum dimensions and electrical parameters for a given frequency response.

\section{REFERENCES}

[1] I. Ladabaum, X. Jin, H. T. Soh, A. Atalar, and B. T. KhuriYakub, "Surface micromachined capacitive ultrasonic transducers," IEEE Trans. Ultrason., Ferroelect., Freq. Contr., vol. 45, pp. 678-690, 1998.

[2] A. Bozkurt, I. Ladabaum, A. Atalar, and B. T. Khuri-Yakub, "Theory and analysis of electrode size optimization for capacitive microfabricated ultrasonic transducers," IEEE Trans. Ultrason., Ferroelect., Freq. Contr., vol. 46, pp. 1364-1374, 1999.

[3] G. G. Yaralioglu, M. H. Badi, A. S. Ergun, and B. T. Khuri-Yakub, "Improved equivalent circuit and finite element method modelling of capacitive micromachined ultrasonic transducers," in Proc. of 2003 Ultrasonics Symposium, pp. 469-472, 2003.

[4] S. Olcum, M. N. Senlik, and A. Atalar, "Optimization of the gainbandwidth product of capacitive micromachined ultrasonic transducers," to be published in IEEE Trans. Ultrason., Ferroelect., Freq. Contr., 2005.

[5] A. Lohfink, P. C. Eccardt, W. Benecke, and M. Meixner, "Derivation of a 1D cMUT model from FEM results for linear and nonlinear equivalent circuit simulation," in Proc. of 2003 Ultrasonics Symposium, pp. 465468, 2003.

[6] C. Bayram, S. Olcum, M. N. Senlik, and A. Atalar, "Bandwidth improvement in a cMUT array with mixed sized elements," in Proc. of 2005 Ultrasonics Symposium, 2005.

[7] A. Nikoozadeh, B. Bayram, G. G. Yaralioglu, and B. T. Khuri-Yakub, "Analytical calculation of collapse voltage of cMUT membrane," in Proc. of 2004 Ultrasonics Symposium, pp. 256-259, 2004.

[8] D. Pozar, Microwave Engineering. New York: John Wiley and Sons, 1998. 